EXTENDED REPORT

\title{
Is DAS28 an appropriate tool to assess remission in rheumatoid arthritis?
}

\author{
H Mäkinen, H Kautiainen, P Hannonen, T Sokka
}

Ann Rheum Dis 2005;64:1410-1413. doi: 10.1136/ard.2005.037333

See end of article for authors' affiliations

....................

Correspondence to: Dr H Mäkinen, Jyväskylä Central Hospital, Keskussairaalantie 19, 40620 Jyväskylä, Finland; heidi.makinen@kshp.fi

Accepted 2 June 2005 Published Online First 7 June 2005

\begin{abstract}
Objectives: To study which cut off point of DAS28 corresponds to fulfilment of the American Rheumatism Association (ARA) preliminary remission criteria, and clinical remission criteria in patients with rheumatoid arthritis (RA).

Methods: All adult patients diagnosed with RA at Jyväskylä Central Hospital 1997-98 were assessed for remission at 5 years. Remission was defined as (a) ARA remission; (b) clinical remission (defined as no tender or swollen joints and normal erythrocyte sedimentation rate). DAS28 was used to measure disease activity. A receiver operating characteristics curve analysis was performed to calculate a cut off point of DAS28 that best corresponds to the ARA remission criteria and the clinical remission criteria.

Results: 161 patients (mean age 57 years, $107(66 \%)$ female, $98(61 \%)$ with positive rheumatoid factor, and $51(32 \%)$ with erosions) were studied. At 5 years, 19 (12\%) patients met the ARA remission criteria, and 55 (34\%) met the clinical remission criteria. The cut off value of DAS28 was 2.32 for the ARA remission criteria, and 2.68 for the clinical remission criteria. In patients with DAS28 <2.32, 11/57 (19\%) had tender joints, 6/ 57 (11\%) had swollen joints, and 4/57 (7\%) had both tender and swollen joints (66 joint count).

Conclusion: In this study the DAS28 cut off point for the ARA remission was lower than in previous studies. The cut off point for DAS28 remission remains controversial. A substantial proportion of patients below the DAS28 cut off point for remission have tender or swollen joints, or both. DAS28 may not be an appropriate tool for assessment of remission in RA.
\end{abstract}

$\mathrm{T}$ he goal of treatment of rheumatoid arthritis (RA) is remission. Definition of remission, however, remains controversial. The preliminary remission criteria for RA proposed by Pinals et al in $1981^{1}$ were adopted by the American Rheumatism Association (ARA). Other definitions such as "being symptom-free" or "no arthritis on examination" have also been used..$^{2-8}$

The Disease Activity Score (DAS) ${ }^{9-11}$ and its modified version including 28 joint count (DAS28) ${ }^{12}$ were developed to measure disease activity in patients with RA. In patients with early RA, Prevoo et al found that DAS $<1.6$ best correlates with the ARA remission criteria. ${ }^{13}$ Later the corresponding cut off point of $<2.6$ for DAS28 was derived from a formula developed to convert DAS to DAS28. ${ }^{14}$ Recently, even higher DAS28 cut off points of 2.81 and 2.66 have been reported to correspond to the ARA remission criteria in two separate patient populations. ${ }^{15}{ }^{16}$ Both DAS $<1.6$ and DAS28 $<2.6$ have been used in studies to define remission in RA. ${ }^{17-19}$

Discussion has arisen about the validity of DAS28 for assessing remission. ${ }^{20} 21$ Therefore, we aimed at studying which cut off point of DAS28 corresponds to fulfilment of the ARA remission criteria, and a simple set of clinical remission criteria (defined as no tender or swollen joints and normal erythrocyte sedimentation rate (ESR)). The positive predictive value of each individual ARA remission criterion was examined. Furthermore, we calculated the percentage of patients with the DAS28 remission who had swollen or tender joints, or both.

\section{PATIENTS AND METHODS}

Study population

Jyväskylä Central Hospital is the only rheumatology centre in the Central Finland District and serves a population of 265 000. All new patients with RA are referred to the hospital for diagnosis and start of treatment. All 237 patients with recent onset inflammatory arthritis, older than 16 years who did not meet criteria or show clinical signs of other specific arthritides (crystal deposit disease, reactive arthritis, ankylosing spondylitis, psoriatic arthritis, etc) were included in the RA 1997-98 inception cohort. These patients received rheumatology care at Jyväskylä Central Hospital for at least 2 years after the diagnosis by a multidisciplinary team and subsequently were invited to participate in a 5 year study visit. A total of 161 patients who cumulatively fulfilled the ARA criteria for RA were included in this study.

\section{Treatment strategies}

Disease modifying antirheumatic drugs were started at the time of diagnosis. The target of treatment was clinical remission.

\section{Clinical and laboratory assessments}

Measures at baseline and at 5 years included 68 tender and 66 swollen joint counts ${ }^{22}$; laboratory tests, including ESR, C

Table 1 Patient characteristics of 161 patients with RA at baseline

\begin{tabular}{ll}
\hline Age (years), mean (range) & $57(22-85)$ \\
$\begin{array}{l}\text { Female, No (\%) } \\
\text { Duration of symptoms before diagnosis (months), } \\
\text { median (IQR) }\end{array}$ & $5(3-11)$ \\
RF positive ever, No (\%) & $98(61)$ \\
Met ARA 1987 criteria for RA, No (\%) & $152(94)$ \\
Patients with erosions, No (\%) & $51(32)$ \\
\hline
\end{tabular}

Abbreviations: ARA, American Rheumatism Association; $\mathrm{Cl}$, confidence interval; DAS28, 28 joint count Disease Activity Score; ESR, erythrocyte sedimentation rate; $\mathrm{GH}$, general health; RA, rheumatoid arthritis; ROC, receiver operating characteristic; VAS, visual analogue scale 
reactive protein, and rheumatoid factor; self reported pain and global health on a $100 \mathrm{~mm}$ visual analogue scale (VAS), functional capacity according to the Health Assessment Questionnaire, self reported morning stiffness in minutes; and radiographs of the hands and feet. The date of starting and stopping of each disease modifying antirheumatic drug was recorded.

\section{Definition of remission}

We used two separate sets of criteria to define remission at 5 years. The ARA remission criteria require $(a)$ no joint or tendon sheath swelling; $(b)$ no joint tenderness; $(c)$ normal ESR of $<20 \mathrm{~mm} / \mathrm{lst} \mathrm{h}$ in men and $<30 \mathrm{~mm} / \mathrm{lst} \mathrm{h}$ in women; $(d)$ morning stiffness $\leqslant 15$ minutes; and $(e)$ no history of joint pain (VAS $\leqslant 10 \mathrm{~mm}$ on a scale of $1-100 \mathrm{~mm}$ ). ${ }^{23}$ The requirement of fatigue was excluded, but all the other five criteria had to be fulfilled. Because in previous studies only four of the five ARA remission criteria have been required for remission (fatigue excluded), we tested that set of the ARA criteria also. We did not require a 2 month period of follow up. Clinical remission was defined as $(a)$ no tender joints; $(b)$ no swollen joints; and (c) normal ESR of $<20 \mathrm{~mm} / \mathrm{lst} \mathrm{h}$ in men and $<30 \mathrm{~mm} / \mathrm{lst} \mathrm{h}$ in women.

\section{Disease activity score (DAS28)}

DAS28 is a validated index of RA disease activity. It consists of four measures: 28 tender (TJC28) and swollen joint (SJC28) counts, ESR, and patients general health (GH) measured on a $100 \mathrm{~mm}$ VAS. ${ }^{12}$ It was calculated using the formula ${ }^{12}$ :

$0.56 \times \sqrt{ }(\mathrm{TJC} 28)+0.28 \times \sqrt{ }(\mathrm{SJC} 28)+0.70 \times \ln (\mathrm{ESR})+0.014 \times(\mathrm{GH})$

\section{Statistical methods}

Receiver operating characteristic (ROC) curves were constructed to determine the cut off point of DAS28 that corresponds to the ARA remission criteria and to the clinical remission criteria, with bias corrected accelerated bootstrap confidence intervals (CIs).

Sensitivity, specificity, positive predictive value, likelihood ratio, and their 95\% CI values were calculated for each remission criterion.

\section{RESULTS}

A total of 196 patients from 237 patients with RA diagnosed in 1997-98 at Jyväskylä Central Hospital participated in the 5 year study visit (20 patients had died, seven patients refused to take part, four patients were too sick to participate, five patients had moved, and the diagnosis of five patients had changed). A total of 161 of these patients cumulatively fulfilled the ARA criteria for RA and were included in the analysis. Table 1 shows the characteristics of the 161 patients with RA at baseline.

At 5 years, $19(12 \%(95 \%$ CI $7 \%$ to $18 \%))$ of the 161 patients with RA met the ARA remission criteria, including $106(66 \%)$ with no swollen joints, 69 (43\%) with no tender joints, 119 (74\%) with a normal ESR, 65 (40\%) with morning stiffness $\leqslant 15$ minutes, and $32(20 \%)$ patients with no pain (table 2). The positive predictive value for the ARA remission was lowest for normal ESR ( $16 \%$ ), and highest for no history of joint pain (56\%). Similarly, the likelihood ratio was lowest for normal ESR (1.40) and highest for no joint pain (10.00) (table 2). According to the less rigorous ARA remission criteria (four of the five ARA remission criteria had to be fulfilled and fatigue was excluded) 40 (25\% (95\% CI 19\% to $33 \%)$ ) of the patients were in remission. A total of 55 patients (34\% (95\% CI $27 \%$ to $42 \%)$ ) met the simple set of clinical remission criteria at 5 years.

Figure 1 shows the ROC curves of DAS28 when used to define the presence or absence of remission using the ARA

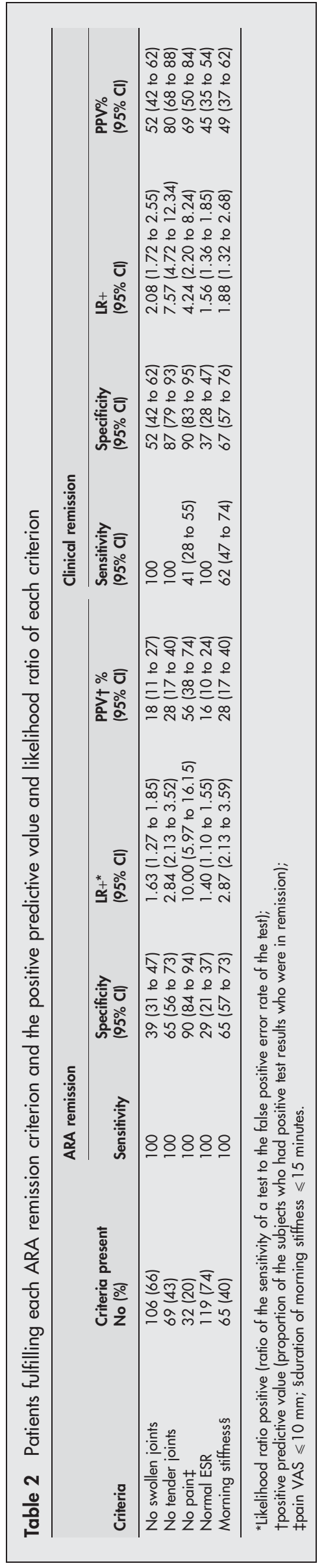




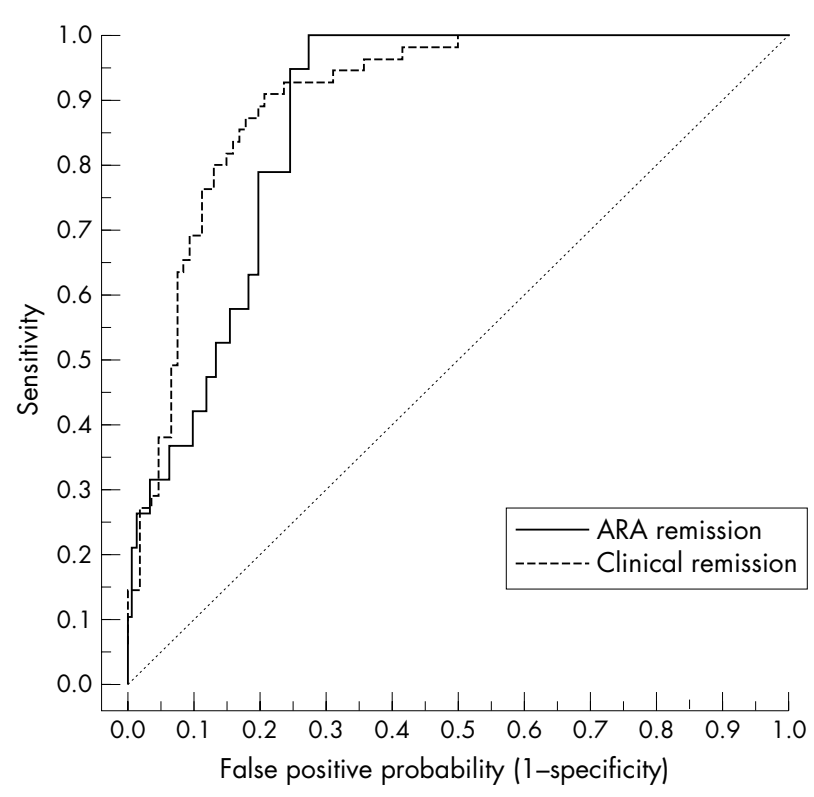

Figure 1 ROC curves of DAS28 when used to define the presence or absence of the ARA remission criteria and the clinical remission criteria.

criteria (fatigue was excluded and all the other five criteria had to be fulfilled), and the clinical remission criteria. The area under the ROC curves was 0.87 (95\% CI 0.82 to 0.93 ) for the ARA remission criteria and 0.90 (95\% CI 0.84 to 0.94 ) for the clinical remission criteria and 0.89 (95\% CI 0.83 to 0.94 ) for the less rigorous ARA remission criteria (table 3 ).

The cut off value for DAS28 was 2.32 (sensitivity 100\%, specificity $73 \%$ ) for the ARA remission criteria (fatigue excluded), 2.60 (sensitivity 93\%, specificity $76 \%$ ) for the less rigorous set of ARA remission criteria (fatigue excluded and four of the remaining five criteria had to be fulfilled), and 2.68 (sensitivity 91\%, specificity $79 \%$ ) for the clinical remission criteria (table 3 ).

Of the 57 patients who had DAS28 <2.32, five (9\%) patients had tender joints, four $(7 \%)$ had swollen joints, and two $(4 \%)$ had both tender and swollen joints on the 28 joint count. On the 66 joint count the corresponding figures were $11(19 \%)$, six $(11 \%)$, and four $(7 \%)$ (table 4$)$. If we had used the previously proposed cut off point of 2.6 as the limit of DAS28 remission in our cohort, a higher proportion of our patients $(66(41 \%))$ would have been included in the remission group. Of these 66 patients, $15(23 \%)$ had tender and $6(9 \%)$ swollen joints, and $4(6 \%)$ had both tender and swollen joints (66 joint count) (table 4$)$.

\section{DISCUSSION}

In this study, DAS28 $<2.32$ corresponded to the fulfilment of the ARA remission criteria (five of the ARA remission criteria had to be fulfilled, fatigue was excluded) and DAS28 $<2.68$ corresponded to the clinical remission criteria defined as no tender and no swollen joints and normal ESR. We also calculated the cut off point of DAS28 using a less rigorous set of ARA remission criteria which were used by Balsa et al ${ }^{15}$ and Fransen $e{ }{ }^{16}{ }^{16}$ in their studies, and the cut off point was 2.6 in agreement with the study of Fransen et al. An even higher cut off point of DAS28 2.81 for this set of ARA remission criteria was reported by Balsa et al. ${ }^{15}$

The preliminary RA remission criteria of Pinals et al ${ }^{1}$ require that five of the six criteria are fulfilled. The criteria explicitly accept that patients with either tender or swollen joints can be considered to be in remission, although patients are not allowed to have both tender and swollen joints. In our study $7 \%$ of the patients who were in DAS28 remission (DAS28 $<2.32$ ) had both tender and swollen joints. Furthermore, as many as $19 \%$ of patients had tender joints and 11\% had swollen joints. Most rheumatologists, however, would agree that patients with swollen and/or tender joints are not in true clinical remission.

Landewe et al compared DAS28 remission with DAS remission and concluded that DAS (DAS $<1.6$ ) remission is far more conservative than DAS28 (DAS28 <2.6) remission. ${ }^{20}$ On the other hand, Fransen et al showed that DAS $<1.6$ and DAS28 $<2.6$ were equal when compared with ARA remission criteria. ${ }^{16}$ Landewe et al suggested that the discrepancy between DAS and DAS28 remission is explained by activity in joints that are not included in the DAS28 (feet and ankles). We agree that DAS28 at a cut off level of 2.6 should not be used to define remission in clinical practice or clinical trials. ${ }^{20}$

The criterion of no joint pain had the highest positive predictive value of the five ARA remission criteria in our study. A total of $56 \%$ of our patients with no joint pain fulfilled the ARA defined remission. On the other hand, ESR had the lowest positive predictive value; only $16 \%$ of the patients with normal ESR fulfilled the ARA defined remission (table 2 ). Our results are in line with those obtained by Balsa et al, who showed that pain has the highest positive predictive value and ESR the lowest. ${ }^{15}$ Therefore, normal ESR in a patient with RA does not tell much about whether he or she is in remission or not. In contrast, when a patient with RA has no pain the chances of fulfilling the ARA defined remission are considerable.

The likelihood ratio of ESR was low, 1.40. Accordingly, the criterion of normal ESR does not improve the accuracy of the ARA remission criteria. Further, minor changes in ESR may change the DAS28 value strikingly. For example, if a patient has two tender and no swollen joints with GH 10 and her/his

\begin{tabular}{|c|c|c|c|}
\hline \multirow[b]{2}{*}{ Characteristics } & \multicolumn{3}{|l|}{ Remission criteria } \\
\hline & $\begin{array}{l}\text { ARA* } \\
(95 \% \text { Cl) }\end{array}$ & $\begin{array}{l}\text { ARA } \\
(95 \% \mathrm{Cl})\end{array}$ & $\begin{array}{l}\text { Clinical } \\
(95 \% \mathrm{CI})\end{array}$ \\
\hline $\begin{array}{l}\text { Cut off point } \\
\text { Area under ROC curvesł } \\
\text { Sensitivity (\%) } \\
\text { Specificity (\%) } \\
\text { PPV§ (\%) } \\
\text { LR+ }\end{array}$ & $\begin{array}{l}2.32 \\
0.87(0.82 \text { to } 0.93) \\
100(82 \text { to } 100) \\
73(64 \text { to } 80) \\
33(21 \text { to } 46) \\
3.64(2.66 \text { to } 4.71)\end{array}$ & $\begin{array}{l}2.60 \\
0.89(0.83 \text { to } 0.94) \\
93(80 \text { to } 98) \\
76(67 \text { to } 83) \\
56(43 \text { to } 68) \\
3.80(2.76 \text { to } 5.32)\end{array}$ & $\begin{array}{l}2.68 \\
0.90(0.84 \text { to } 0.94) \\
91(80 \text { to } 97) \\
79(70 \text { to } 87) \\
69(57 \text { to } 80) \\
4.38(3.05 \text { to } 6.48)\end{array}$ \\
\hline \multicolumn{4}{|c|}{$\begin{array}{l}\text { *ARA remission criteria, all the five criteria must be fulfilled (fatigue excluded); †ARA remission criteria, four of the } \\
\text { five criteria must be fulfilled (fatigue excluded); ‡area under the receiver operating characteristic curves. } 95 \% \\
\text { confidence interval obtained by bias corrected and accelerated bootstrapping ( } 5000 \text { replications); } \$ \text { positive } \\
\text { predictive value (proportion of the subjects who had positive test results who were in remission); } \uparrow \text { likelihood ratio } \\
\text { positive (ratio of the sensitivity of a test to the false positive error rate of the test). }\end{array}$} \\
\hline
\end{tabular}


Table 4 Cut off values of DAS28 and number of patients not fulfilling each individual ARA remission criterion

\begin{tabular}{|c|c|c|c|}
\hline & *DAS28 $<2.32$ & tDAS28 $<2.6$ & fDAS28 $<2.68$ \\
\hline Patients (n) & 57 & 66 & 72 \\
\hline \multicolumn{4}{|l|}{ Patients with tender joints } \\
\hline 28 Joint count & $5(9)$ & $6(9)$ & $7(8)$ \\
\hline 66 Joint count & 11 (19) & $15(23)$ & $17(24)$ \\
\hline \multicolumn{4}{|l|}{ Patients with swollen joints } \\
\hline 28 Joint count & $4(7)$ & $5(8)$ & $5(7)$ \\
\hline 66 Joint count & 6 (11) & $6(9)$ & $6(8)$ \\
\hline \multicolumn{4}{|c|}{ Patients with tender and swollen joints } \\
\hline 28 Joint count & $2(4)$ & $2(4)$ & $2(3)$ \\
\hline 66 Joint count & $4(7)$ & $4(6)$ & $4(6)$ \\
\hline Patients with raised ESR & $2(4)$ & $3(5)$ & $4(6)$ \\
\hline Patients with morning stiffness & $23(40)$ & $25(38)$ & $27(38)$ \\
\hline Patients with joint pain & $33(58)$ & $38(58)$ & $44(61)$ \\
\hline \multicolumn{4}{|c|}{$\begin{array}{l}\text { Results are shown as No (\%). } \\
\text { *The cut off point corresponding to the ARA remission criteria in this study (all the five ARA remission must be } \\
\text { fulfilled and fatigue excluded); the generally accepted cut off point of DAS28 in remission and the cut off point of of } \\
\text { ARA remission in this study when modified ARA criteria are used (four of the five ARA remission criteria must be } \\
\text { fulfilled and fatigue excluded); fthe cut off point corresponding to the clinical remission criteria defined as no } \\
\text { tender or swollen joints and normal ESR. }\end{array}$} \\
\hline
\end{tabular}

ESR rises from 2 to 20 (ESR is normal in both cases), DAS28 rises from 1.41 to 3.02 .

In this study the DAS28 remission cut off point for the ARA remission was lower than in previous studies because we used the ARA remission criteria more strictly, and remains controversial. The small sample size is a limitation of our study and the results need to be confirmed in other studies. The DAS28 reflects disease activity, but it may not be an optimal tool for assessment of remission in RA. All remission criteria so far have included the domain of signs and symptoms of inflammation, whereas the other two major domains of RA, physical function and structural joint damage, have received less attention. ${ }^{24-26}$ We still lack a good definition of remission for daily clinical needs as well as for investigational purposes.

\section{Authors' affiliations}

H Mäkinen, P Hannonen, T Sokka, Jyväskylä Central Hospital, Keskussairaalantie 19, 40620 Jyväskylä, Finland

H Kautiainen, Rheumatism Foundation Hospital, Heinola, Finland T Sokka, Vanderbilt University Medical School, 203 Oxford House, Nashville, TN 37232-4500, USA

\section{REFERENCES}

1 Pinals RS, Masi AT, Larsen RA. Preliminary criteria for clinical remission in rheumatoid arthritis. Arthritis Rheum 1981;24:1308-15.

2 Harrison B, Symmons D. Early inflammatory polyarthritis: results from the Norfolk Arthritis Register with a review of the literature. II. Outcome at three years. Rheumatology (Oxford) 2000;39:939-49.

3 Quinn MA, Green MJ, Marzo-Ortega H, Proudman S, Karim Z, Wakefield RJ et al. Prognostic factors in a large cohort of patients with early undifferentiated inflammatory arthritis after application of a structured management protocol. Arthritis Rheum 2003;48:3039-45.

4 Nissila M, Isomaki H, Kaarela K, Kiviniemi P, Martio J, Sarna S. Prognosis of inflammatory joint diseases. A three-year follow-up study. Scand I Rheumatol 1983;12:33-8.

5 Harrison BJ, Symmons DP, Brennan P, Barrett EM, Silman AJ. Natural remission in inflammatory polyarthritis: issues of definition and prediction. Br J Rheumatol 1996;35:1096-100.

6 Wolfe F, Ross K, Hawley DJ, Roberts FK, Cathey MA. The prognosis of rheumatoid arthritis and undifferentiated polyarthritis syndrome in the clinic: a study of 1141 patients. J Rheumatol 1993;20:2005-9.

7 Eberhardt K, Fex E. Clinical course and remission rate in patients with early rheumatoid arthritis: relationship to outcome after 5 years. $\mathrm{Br} J$ Rheumatol 1998; $37: 1324-9$

8 Pease CT, Bhakta BB, Devlin J, Emery P. Does the age of onset of rheumatoid arthritis influence phenotype? : a prospective study of outcome and prognostic factors, Rheumatology (Oxford) 1999;38:228-34.

9 van der Heijde DM, van 't Hof MA, van Riel PL, Theunisse LA, Lubberts EW, van Leeuwen $M A$, et al. Judging disease activity in clinical practice in rheumatoid arthritis: first step in the development of a disease activity score. Ann Rheum Dis 1990;49:916-20.

10 Fuchs HA. The use of the disease activity score in the analysis of clinical trials in rheumatoid arthritis. J Rheumatol 1993;20:1863-6.

11 van der Heijde DM, van 't Hof M, van Riel PL, van de Putte LB. Development of a disease activity score based on judgment in clinical practice by rheumatologists. J Rheumatol 1993:20:579-81.

12 Prevoo ML, van ' $t$ Hof MA, Kuper HH, van Leeuwen MA, van de Putte $L B$, van Riel PL. Modified disease activity scores that include twenty-eight-joint counts. Development and validation in a prospective longitudinal study of patients with rheumatoid arthritis. Arthritis Rheum 1995;38:44-8.

13 Prevoo ML, van Gestel AM, van ' $\uparrow$ Hof MA, van Rijswijk MH, van de Putte LB, van Riel PL. Remission in a prospective study of patients with rheumatoid arthritis. American Rheumatism Association preliminary remission criteria in relation to the disease activity score. Br J Rheumatol 1996;35:1101-5.

14 van Riel PL, van Gestel AM. Clinical outcome measures in rheumatoid arthritis. Ann Rheum Dis 2000;59(suppl 1):i28-31.

15 Balsa A, Carmona L, Gonzalez-Alvaro I, Belmonte MA, Tena X, Sanmarti R. Value of Disease Activity Score 28 (DAS28) and DAS28-3 compared to American College of Rheumatology-defined remission in rheumatoid arthritis. J Rheumatol 2004:31:40-6.

16 Fransen J, Creemers MC, Van Riel PL. Remission in rheumatoid arthritis: agreement of the disease activity score (DAS28) with the ARA preliminary remission criteria. Rheumatology (Oxford) 2004;43:1252-5.

17 Svensson B, Schaufelberger C, Teleman A, Theander J. Remission and response to early treatment of RA assessed by the Disease Activity Score. BARFOT study group. Better Anti-rheumatic Farmacotherapy. Rheumatology (Oxford) 2000;39:1031-6.

18 Svensson B, Ahlmen M, Forslind K. Treatment of early RA in clinical practice: a comparative study of two different DMARD/corticosteroid options. Clin Exp Rheumatol 2003;21:327-32

19 Breedveld FC, Kavanaugh AF, Cohen SB, Pavelka K, Van Vollenhoven R, Perez JL, et al. Early treatment of rheumatoid arthritis (RA) with adalimumab (Humira) plus methotrexate vs. adalimumab alone or methotrexate alone: the PREMIER Study, Arthritis Rheum 2004;50(suppl): 188.

20 Landewe R, van der Hejde D, Voskouyl A, Moers M. Condensed 28-joint counts jeopardise the construct validity of DAS28-remission: a comparison with the original DAS. Arthritis Rheum 2004;50(suppl):456.

21 van der Heijde DM, Jacobs JW. The original "DAS" and the "DAS28" are not interchangeable: comment on the articles by Prevoo et al. Arthritis Rheum 1998:41:942-5.

22 Felson DT, Anderson JJ, Boers M, Bombardier C, Chernoff M, Fried B, et al. The American College of Rheumatology preliminary core set of disease activity measures for rheumatoid arthritis clinical trials. The Committee on Outcome Measures in Rheumatoid Arthritis Clinical Trials. Arthritis Rheum 1993;36:729-40

23 Sokka T, Pincus T. Most patients receiving routine care for rheumatoid arthritis in 2001 did not meet inclusion criteria for most recent clinical trials or american college of rheumatology criteria for remission. I Rheumatol 2003;30:1138-46.

24 Paulus HE. Defining remission in rheumatoid arthritis: what is it? Does it matter? J Rheumatol 2004:31:1-4.

25 Makinen $\mathrm{H}$, Kautiainen $\mathrm{H}$, Hannonen P, Sokka T. Frequency of remissions in early rheumatoid arthritis defined by 3 sets of criteria. A 5-year followup study. J Rheumatol 2005;32:796-800.

26 Jantti J, Kaarela K, Kautiainen H, Isomaki H, Aho K. Radiographic remission in seropositive rheumatoid arthritis. A 20-year follow-up study. Clin Exp Rheumatol 2001;19:573-6. 